\title{
Low Power LoRa Transmission in Low Earth Orbiting Satellites
}

\author{
Rifath Shaarook ${ }^{1}$ and Dr. Srimathy Kesan ${ }^{2}$ \\ 1,2 Space Kidz India
}

\begin{abstract}
In the recent past, a modulation technique called "LoRa" has become popular in the communication industry due to its long range capabilities with low power consumption, a few satellites have carried LoRa and worked successfully inspite of being skeptical about Doppler shift, packet loss, etc. This paper discusses the preliminary results of LoRa communications established from a recently launched cubesat. Satish Dhawan Satellite (SD Sat) is a 3U Cubesat built by Space kidz India, weighing $1.9 \mathrm{~kg}$, it carried a radiation counter and magnetometer as its payload. It carried a LoRa transmitter which transmits beacon at $100 \mathrm{~mW}$. It is also transmitting FSK RTTY and CW RTTY. The satellite was launched into sunsynchronous orbit at $510 \times 498 \mathrm{~km}$. Multiple ground stations around the world have confirmed the signal reception.
\end{abstract}

Keywords. Cubesat, LoRa, Satellites, LEO, IoT

\section{Introduction}

Space Kidz India is an organization from India working towards promoting Space Education and awareness among the students across the globe. Space Kidz India has previously worked on 12 high-altitude balloon missions to test various subsystems for cubesats at near space environment. It has also successfully completed two suborbital space flights, SKISAT and KALAMSAT which tested the subsystems apart from launching an orbital satellite "KalamSAT V2" launched through ISRO on PSLV C44 in 2019. Satish Dhawan SAT (SDSat) is the second satellite built by students at Space Kidz India, named after one if the founding fathers of India's space agency Indian Space Research Organization, as part of his centenary birth celebration. The aim of the mission was to test the various aspects of the newly designed satellite which was indigenously fabricated end to end. One of the experiment was to test how the low power transmission in LoRa mode would perform. Other experiments includes measuring the ionizing radiation experienced by the satellite from various sources like cosmic radiation, solar flares, etc. and testing how the magnetometer present in the satellite would perform. The team intends to use the magnetometer data for developing an Attitude Control System for cubesats in the future. The satellite's engineering model and flight model are shown in the Figure 1. Both the models are identical and the engineering model is used for all sorts of testing like vibration testing, thermovaccum testing and shock testing while the flight model will the satellite which actually gets to fly onboard the rocket and reaches the orbit. You can also see that the solar panels are attached outside for the power

1,2 Rifath Shaarook, Dr. Srimathy Kesan are with Space Kidz India, Chennai, India, e-mail: cto@skilabz.in, e-mail: ceo@spacekidzindia.in 
generation. Figure 2 shows the internals of the satellite where it is covered with Multi Layer Insulation material for thermal insulation to maintain an operatable temperature inside the satellite.

The satellite is a $3 \mathrm{U}$ cubesat measuring $340 \mathrm{~mm} \times 100 \mathrm{~mm} \times 100 \mathrm{~mm}$ and weighing 1.9 kg. [1] It was launched onboard ISRO's PSLV-C51 launch vehicle on 28th February 2021. Following the launch, the satellite was deployed from the cubesat deployer at 12.15 PM IST over Madagascar. The satellite was inserted into a $510 \mathrm{~km}$ x $498 \mathrm{~km}$ sunsynchnorous orbit with an inclination of 97.5 degrees, making it possible for the satellite to cover the entire earth in the subsequent orbits. After about eight and half hours post the launch, first FSK signals were confirmed over Australia by SatNOGS Network [2] and one of the first LoRa signals were received over USA by an amateur radio operator

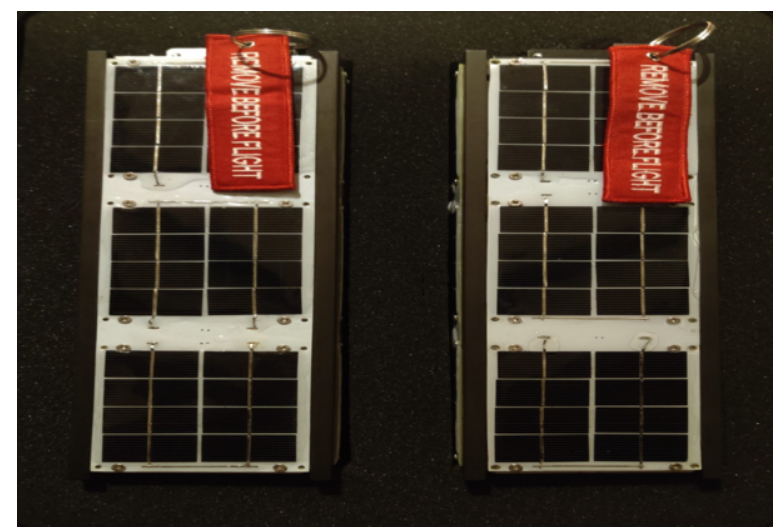

known as N6RFM by his call sign [3].

Figure 1. Engineering and Flight Model of the SDSat

Before the launch the satellite has to be integrated with the launch vehicle, Figure 2 shows the satellite getting integrated into the deployer which will be further attached with the rocket and Figure 3 shows the satellite getting deployed into orbit from the rocket.

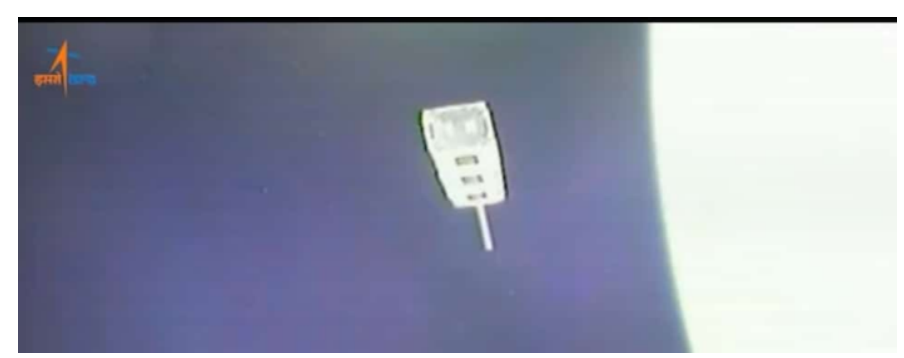

Figure 2. SDSat getting inserted into the deployer 


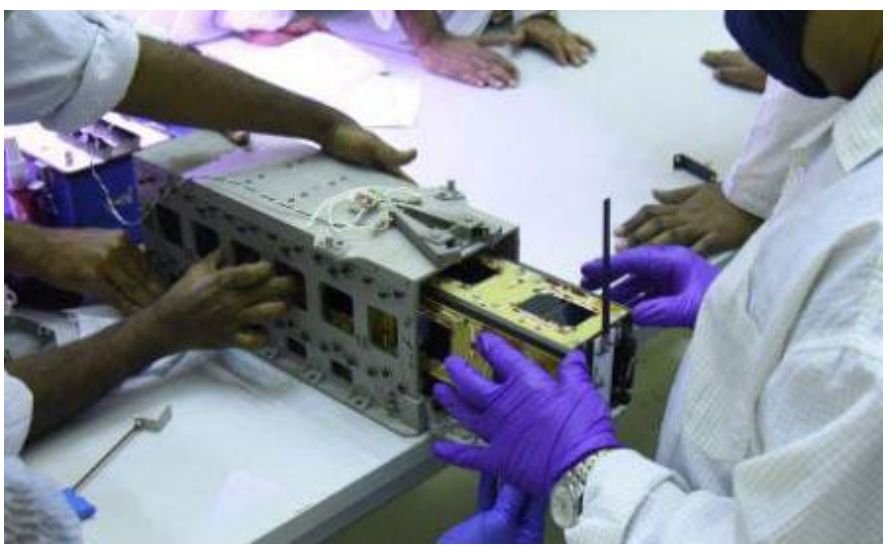

Figure 3. SDSat getting inserted into the deployer

The satellite uses a custom transceiver built using SX1278 from Semtech as it's heart for sending telemetry and receiving telecommands. SX1278 and it's family of chips can transmit in both FSK and LoRa, since LoRa was an experimental feature in this mission, another primary mode of transmission was required and FSK was selected as it has a long heritage of usage in space. The telemetry includes both health data like battery levels, solar panel voltage, etc and sensor data from the main payloads. The transmitter also transmits $\mathrm{CW}$ beacon as it is easier to locate the satellite from ground and perform Doppler calculations. The transceiver carries a single SX1278 chip, thus it operates in half duplex mode, transmission and reception both share the same frequency of 435.5 MHz. Transceiver parameters are given in Table 1.

Table 1. Transceiver Parameters

\begin{tabular}{cc}
\hline Tx Frequency & \multicolumn{1}{c}{$435.5 \mathrm{MHz}$} \\
Rx Frequency & \multicolumn{1}{c}{$435.5 \mathrm{MHz}$} \\
Antenna Type & Tape Measure Dipole \\
Antenna Gain & $2.15 \mathrm{dbi}$ \\
Radiation Pattern & \\
EIRP & Omnidirectional \\
& \\
\end{tabular}




\section{LoRa Transmission}

LoRa is a type of modulation technique developed and patented by Semtech Corporation based in USA and it has become quite popular in the recent days due to its long range transmission capabilities by using small amount of power compared to other types of modulations. [4] LoRa's spread spectrum modulation techniques are based on chirp spread spectrum technology. Since spacecrafts have limited power production capability in space, saving power and making de- vices efficient are one of the most important thing in designing spacecrafts and recent LoRa developments have attracted many teams to use LoRa in their satellites. [5] As LoRa is a favourable technique for IoT systems, many companies intend using space based LoRa gateways for enabling IoT networks, but due to the effect of Doppler shift produced by high orbital velocity in lower earth orbit and wide bandwidth of LoRa modulation has made many skeptical about the capabilities of using LoRa with low cost ground equipment and antenna systems. Parameters of the LoRa transmissions are given in the Table 2 and antenna in its deployed form is given in the Figure 4.

Table 2. LoRa Parameters

\begin{tabular}{cc}
\hline Carrier Frequency & $435.5 \mathrm{MHz}$ \\
Bandwidth & $125 \mathrm{KHz}$ \\
Spreading Factor & 7 \\
Coding Rate & $100 \mathrm{~mW} / 20 \mathrm{dam}$ \\
Output Power & \\
Preamble Length & $8 \mathrm{Symbols}$ \\
Sync Word & 12 \\
Gain & 0
\end{tabular}

An Atmel ATMEGA-32u4 micro controller has been con- nected with SX1278 via SPI interface and it acts as a data handler and communication system manager while the main onboard computer is an Atmel ATMEGA-328.

Antenna is a half-wave dipole and it is made out of simple steel measuring tape. It was cut half the wavelength of the transmitting frequency and tuned using a VNA. Antenna and the transmitter are connected using RG-58 coaxial cable using SMA connectors on both the sides. Threadlocker was used while tightening the SMA to make sure it will not get loosened during the launch due to the vibration and shocks produced. 


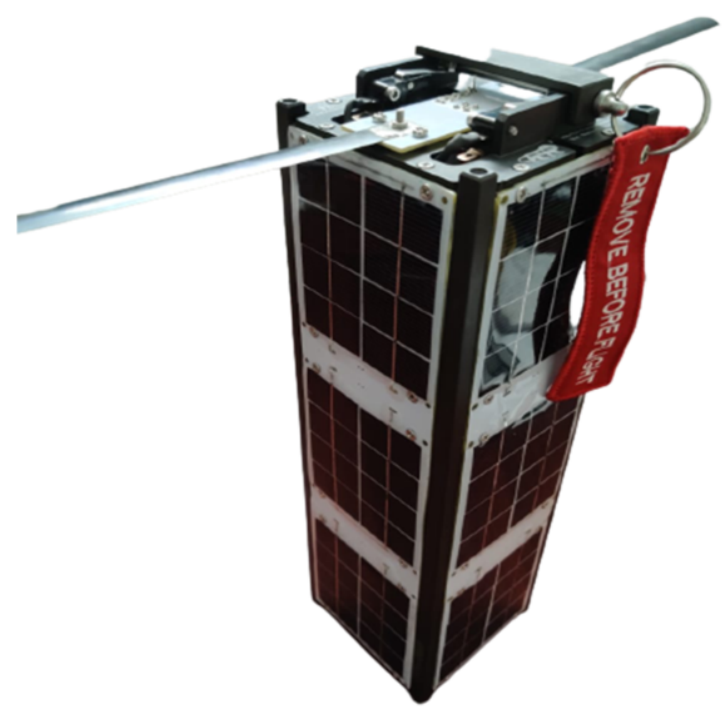

Figure 4. Antenna of the Satellite when it's deployed

The satellite first transmits in FSK RTTY at $100 \mathrm{~mW}$ power and it includes health data like battery power, solar panel power production, on-board computer reset counter, etc and it transmits sensor data which includes data from all the payloads. Then the satellite switches to CW mode where it transmits its name first in high power mode which is 100 $\mathrm{mW}$ and then in low power mode which is $20 \mathrm{~mW}$. We did this to study how easily we can receive $20 \mathrm{~mW}$ beacons so that our future missions can operate beacons in that power alone to save power. The last transmission mode is LoRa where it sends a static message with its name and signifying that LoRa is active. There is also a LoRa repeater mode present in the satellite which we are testing right now and it will become available soon where it will be transmitting short messages sent to it by uplink telecommands. The whole process of transmitting in different modes take around 20 seconds and it will switch to receive mode for 15 seconds to receive telecommands sent from the ground. It acts according to the command it receives or else it will continue the same loop once the 15 seconds receive mode is completed.

The satellite is designed to comply with the rules of International Telecommunication Union. Hence, during the receiving period if a particular telecommand with a secret code is been sent, the satellite will go on a receiving mode and stop the transmission on the whole, until you send another telecommand with a secret code to put the satellite back into normal operations. 


\section{LoRa Reception}

The satellite was deployed from the launch vehicle at 12:15 PM IST over Madagascar, the orbit it was inserted into was a $510 \mathrm{~km}$ x $498 \mathrm{~km}$ sun-synchronous orbit. The satellite was turned on immediately after the deployment but transmission was programmed to be disabled due to safety reasons for 30 minutes. Hence, the satellite was turned on after 30 minutes and first beacons of the satellite were heard over Australia in SatNOGS Network and the signals were confirmed using second reception over Argentina. The first signals from LoRa were received by the Ham Radio operator Mr. Bob known by his call sign N6RFM in TinyGS network. TinyGS is an open- source distributed ground station network having multiple ground stations around the world. [6] During the initial period after the launch, it is hard to exactly locate the satellite, people who receive the satellite's signal uses Two-Line element (TLE) for tracking, it is an orbital parameter to identify the position of the satellite. After the launch, this TLE will not be accurate enough to track, but after a few initial signal receptions, the position of the satellite became accurate enough that many people around the Globe started receiving the signals from SDSat. A typical reception of LoRa beacon during nominal operation of the satellite is shown in the Figure 5. The beacon contains the data "SDSAT, LORA ACTIVE:". Once the repeater is activated, this will also contain the messages sent to the satellite.

On the hardware side setup of the reception, You need a good antenna tuned to receive the specific frequency of the satellite with a low noise amplifier. But many have received the signals using just a generic UHF dipole antenna without any LNA. Figure 6 (left) shows a 10 element Yagi Uda antenna with azimuth/elevation rotator and an LNA while figure 6(right) shows a generic UHF dipole antenna without any LNA mounted inside a window of a building. A study about the comparison of antenna setup and signal reception quality is underway.

You also need a receiver setup with antenna, a SX1278 module with any micrcontroller running generic LoRa recep- tion software with the parameters shown in Table 2, would be able to receive the signals. We are using SX1278 with ESP32 Microcontroller running TinyGS software for the reception and the setup of one such hardware with a simple antenna is shown in the Figure 7. It is located in the Robotics Lab of Middlesex University, Dubai where students are receiving our satellite signals. It shows a simple dipole antenna made up of Co-axial cable with a band pass filter and low noise amplifier connected to a ESP32 board known as TTGO which also contains a SX127x LoRa chip and an OLED display for easy operation. How to install the software and setup your own receiving station can be found at the website of TinyGS. 


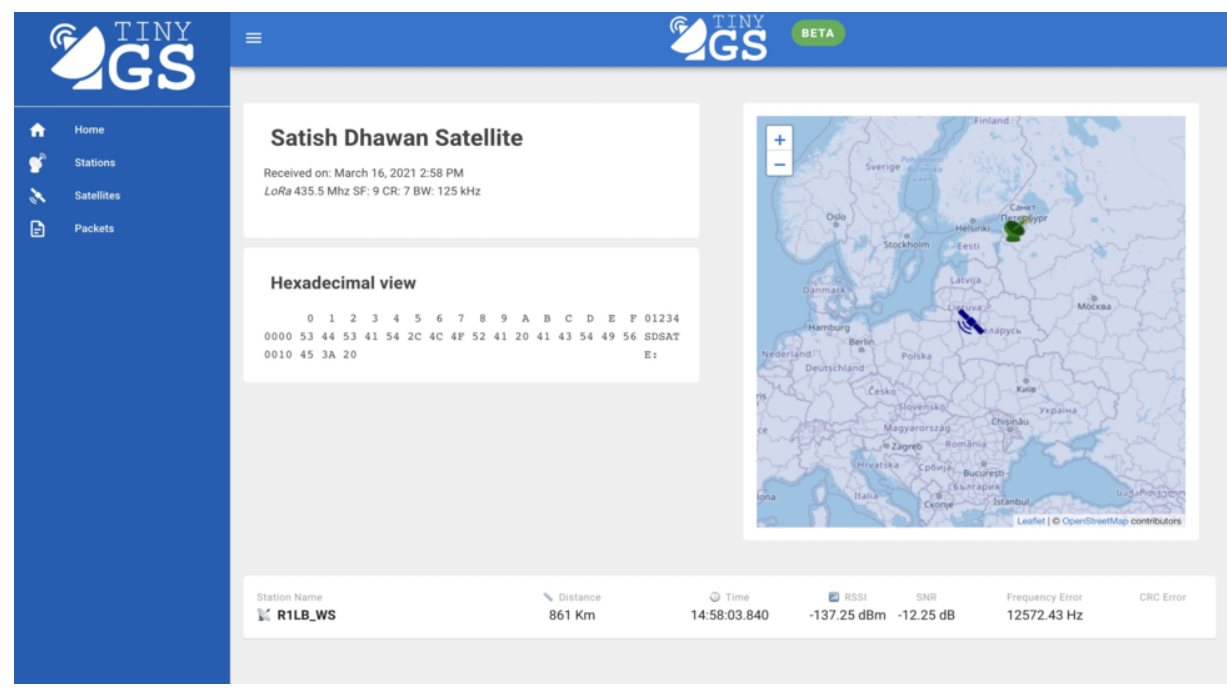

Figure 5. LoRa beacon received in TinyGS Network

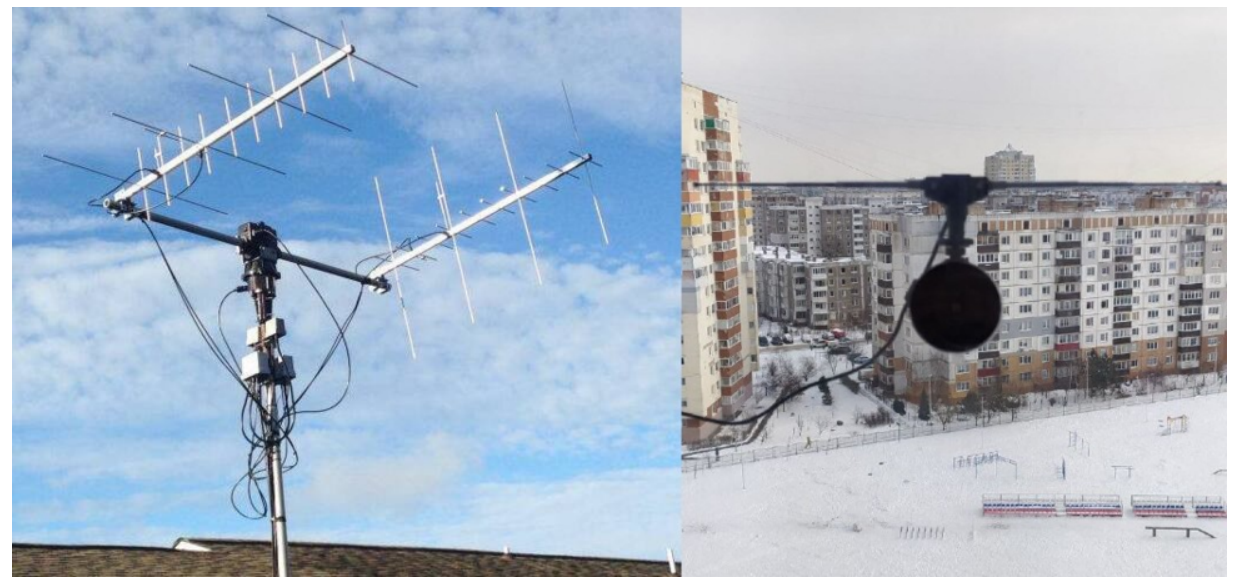

Figure 6. Yagi UDA antenna and simple dipole antenna

\section{Tracking the Satellite}

The satellite is in lower earth orbit, traveling at a velocity of $28,000 \mathrm{~km} / \mathrm{h}$, which means that the satellite will orbit the earth around 16 times a day and cross a given location two times a day. To receive the signals from satellite you should know when to look at the sky and get ready for receiving the satellite so that you can switch on your receiving hardware at that particular time so you can save a lot of electrical power and also be sure that the signals are from your satellite, to know this you can use lot of free and open source software like Orbitron, GPredict, etc. The TLE file of the given satellite can be downloaded from the Celestrak or similar TLE providing website and loaded up 
on the tracking software to find the exact location of the satellite in the orbit at any given moment. There are similar software available for smartphones also, like Satorbit which allows you to track the satellite using your mobile devices. Figure 8 shows the SDSat being tracked using the SatOrbit software in an Android phone.

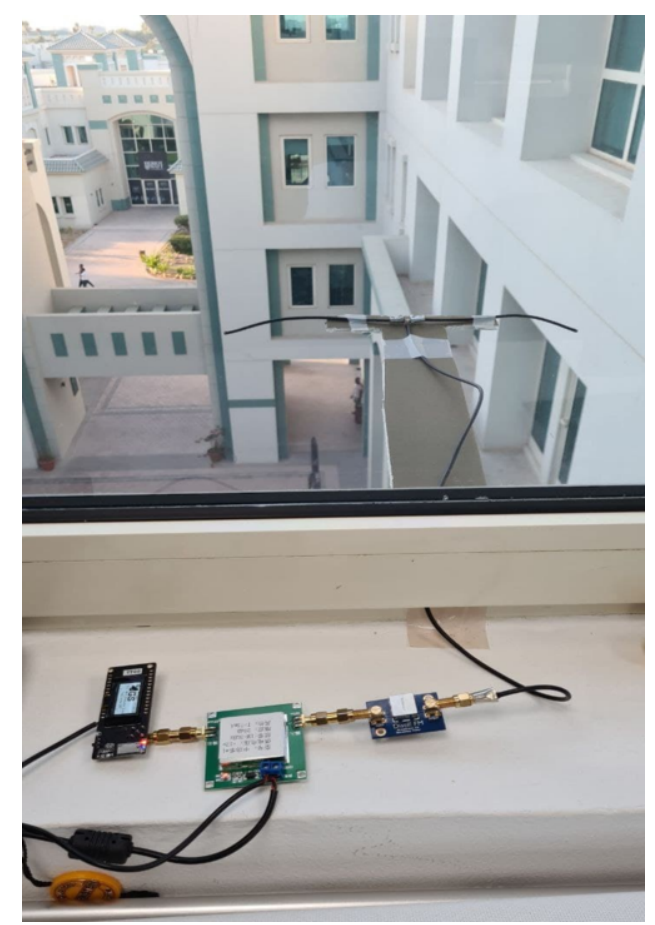

Figure 7. Ground Station setup at the Robotics Lab, Middlesex University, Dubai.

\section{Downlink Signal Waterfall}

The downlink signals of SDSat during nominal operations were received using a Software Defined Radio and various formats of the downlink are noted in the waterfall. Figure 9 shows the SDSat's signal received over US East Coast by amateur radio operator Scott Chapman, known as K4KDR by his callsign. The figure shows a series of data from the bottom and a 15-second interval where the satellite will go to receive mode and wait for any telecommands that user may uplink. Then it transmits it's health data in RTTY format which con- tains data like Satellite's onboard temperature, battery level, solar panel power level, reset counter, special messages from the previous uplinked commands if any. And then the payload data gets transmitted in which it contains data from sensors like Intertial Measurement Unit, Onboard Radiation Counter, etc. Both the RTTY are transmitted with a transmission power of $100 \mathrm{~mW}$. Followed by this, two Morse code beacons are sent which contains the word "SDSAT". First it is transmitted with a power of $100 \mathrm{~mW}$ then with the power of $20 \mathrm{~mW}$. Lastly the satellite transmits LoRa signal with $100 \mathrm{~mW}$ of transmission power. Due to the LoRa's wide bandwidth of $125 \mathrm{KHz}$, it's hard to monitor it on the SDR waterfall but it gets received 
by the LoRa hardware, again it shows the sensitivity and high possibility of reception in long range with LoRa technology.

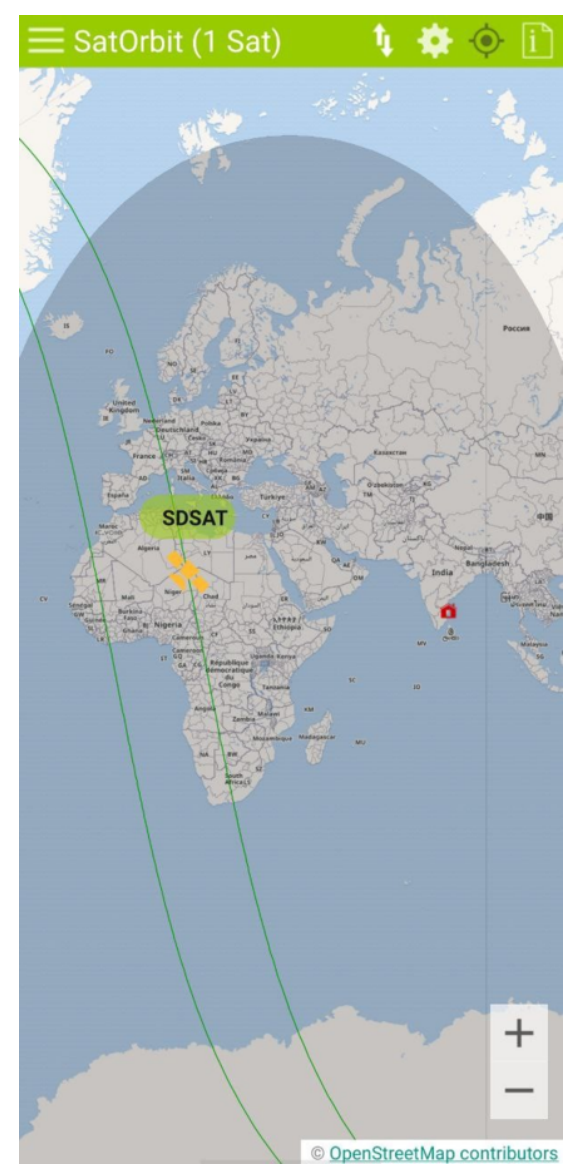

Figure 8. SDSat being tracked in the SatOrbit App

\section{Conclusion}

Initial observations have shown that it is possible to receive $100 \mathrm{~mW}$ LoRa signals from a satellite deployed in low earth orbit. We've also seen successful receptions and decoding of FSK RTTY at $100 \mathrm{~mW}$ and $\mathrm{CW}$ in both $100 \mathrm{~mW}$ and $20 \mathrm{~mW}$ of output power. People have come up with many antenna ideas from simple dipole to complex directional antenna with satellite trackers. More study about the antenna system and reception hardware is underway. 


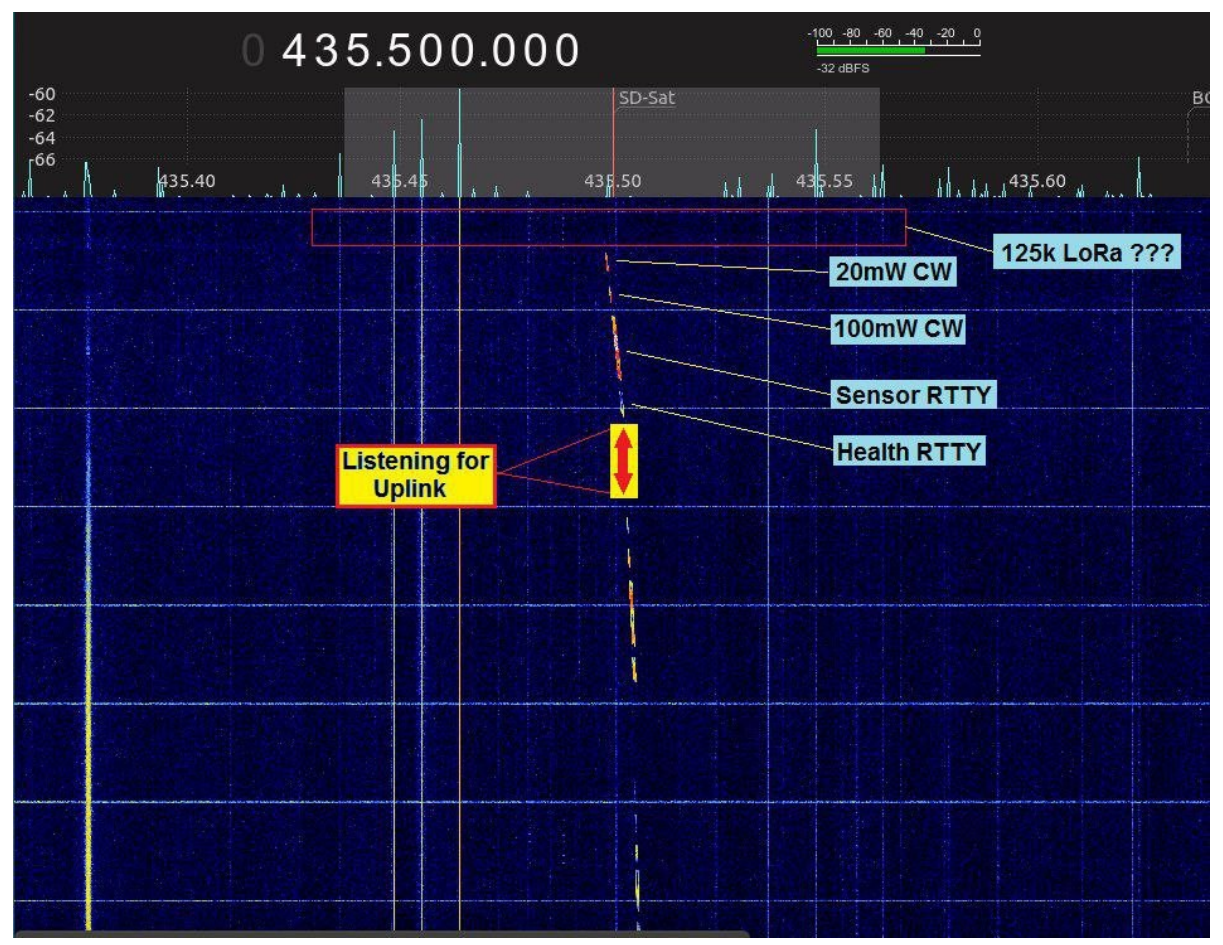

Figure 9. SDSat's signal waterfall received using SDR

\section{Acknowledgement}

Satish Dhawan SAT was supported by the ISRO and IN- SPACe. [7]

\section{References}

1. C. P.S. University, “Cubesat Design Specification.” [Online]. Available: https://www.cubesat.org/cubesatinfo

2. SatNOGS, “Observation \#3703386." [Online]. Available: https://network. satnogs.org/observations/3703386/

3. B. N6RFM, "SDSat First LoRa signals." [Online]. Available: https://twitter.com/n6rfm/status/1366063930829918210

4. Semtech, "What is LoRa?" [Online]. Available: https://www.semtech. com/lora/what-is-lora

5. T. Wu, D. Qu, and G. Zhang, "Research on LoRa Adaptability in the LEO Satellites Internet of Things," IEE Xplore. [Online]. Available: https://ieeexplore.ieee.org/document/8766462

6. "Welcome to TinyGS, the Open Source Global Satellite Network." [Online]. Available: https://tinygs.com/

7. "Satish Dhawan SAT (SDSAT)." [Online]. Available: https://www.isro. gov.in/Spacecraft/satishdhawan-sat-sdsat 